\title{
Elucidation of the Nature of Structural Relaxation in Glassy D-Sorbitol
}

Marcin Krynski ${ }^{1,2 *}$, Felix Mocanu ${ }^{1}$, Stephen Elliott ${ }^{1}$

1. Department of Chemistry, University of Cambridge, Cambridge, UK

\section{Supplementary Information}

\section{VASP parameters}

For the VASP $a b$ initio simulations, the exchange-correlation energy was calculated using the revised Perdew-Burke-Ernzerhof ${ }^{1}$ functional with the Padé approximation. The electronic structure was described using a projector-augmented wave ${ }^{2}$ with a $450 \mathrm{eV}$ energy cut-off, while the Parinello-Rahman (NPT) dynamics were controlled with a Langevin thermostat ${ }^{3,4}$ with a time step of $1 \mathrm{fs}$. The Brillouin zone was sampled at the $\Gamma$ point using a Monkhorst-Pack grid ${ }^{5}$ Van der Waals interactions were approximated with the D3 correction method of Grimme ${ }^{6}$ with BeckeJonson damping ${ }^{7}$. All calculations were performed in a cubic supercell box with periodic boundary conditions. 


\section{Simulation procedure}

A two-stage procedure was implemented for the purpose of this project (presented graphically in Figure S1): initial preparation of amorphous configurations at various temperatures was performed in LAMMPS and generation of the final MD trajectories in VASP.

During the first stage, performed using the LAMMPS code, initial structures of 12 sorbitol molecules were obtained by melting a crystalline model of d-sorbitol using MD simulations at 800 K for 100 ps using the Nosé-Hoover thermostat (NVT), maintaining a density of $1.13 \mathrm{~g} / \mathrm{cm}^{3}$. Next, liquid samples were quenched from $800 \mathrm{~K}$ to $400 \mathrm{~K}$ with a $10 \mathrm{~K} / \mathrm{ps}$ quench rate within the NVT ensemble and on increasing gradually the initial density to $1.34 \mathrm{~g} / \mathrm{cm}^{3}$. At $400 \mathrm{~K}$, an additional 50 ps of MD simulation was carried out within the isothermal-isobaric Langevin barostat (NPT) in order to equilibrate the obtained models. Starting at $400 \mathrm{~K}$, an NPT quench was performed to 80 $\mathrm{K}$ with a $1 \mathrm{~K} / \mathrm{ps}$ quench rate, during which sample configurations were obtained at $20 \mathrm{~K}$ intervals. As the objective of this study is not to investigate the influence of the quench rate on the glassy state, the authors employed a cooling rate matching similar studies ${ }^{8-10}$. Much wider analyses of the quench-rate effect on the glassy phase can be found in ${ }^{11-14}$, where even faster rates than $1 \mathrm{~K} / \mathrm{ps}$ lead to acceptable results. The extracted configurations were then additionally equilibrated for 20 ps within the NPT ensemble. After equilibration, the obtained structures were used as initial configurations for the second stage, where an additional equilibration was performed by conducting 2 ps AIMD (VASP) within the NPT ensemble at all temperatures. 


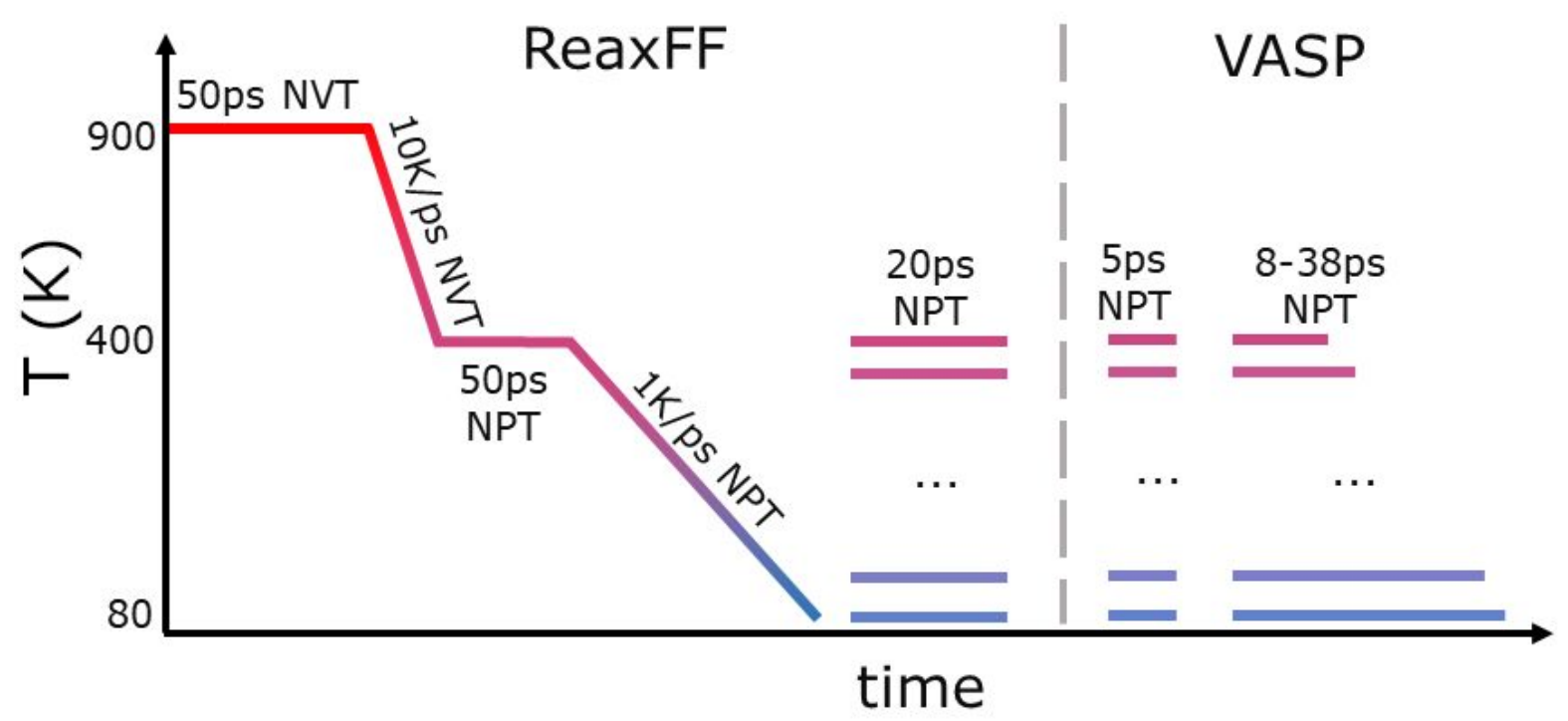

Figure S1 Schematic representation of the simulation procedure. Horizontal and diagonal lines represent MD calculations at different stages.

\section{Validation of the ab initio model}

Figure S2 shows the time evolutions of: a) enthalpy per atom; b) average temperature; and c) pressure, during $2 \mathrm{ps}$ of AIMD equilibration at $300 \mathrm{~K}$. It is evident that none of the presented parameters show any signs of a drift, and the standard-deviation to mean-value ratio stays below $0.1 \%$ for the enthalpy, $5 \%$ for temperature and $1 \%$ for volume. Additionally, the autocorrelation function of the discussed parameters decreases to a value below 0.05 just after around $50 \mathrm{~ns}$, as shown for the case of the temperature in Figure S3. The presented data indicate that thermal equilibrium is achieved during the classical MD equilibration, so the additional $2 \mathrm{ps}$ of ab initio equilibration is not as crucial and plays the role of an additional precaution. It is worth noting that similar equilibrium tests were performed not only for the AIMD equilibration step but also for the production data, and no signs indicating states out of equilibrium were found. 
Lastly, a total of $1 \mathrm{~ns}$ of AIMD trajectories were obtained, where the computational time was distributed according to an exponential distribution, i.e. 8 ps at $400 \mathrm{~K}$ and $38 \mathrm{ps}$ at $80 \mathrm{~K}$. This was done to ensure fairly equal statistics for the calculation of observed phenomena over all studied temperatures.

The data that support the findings of this study are available in the Apollo - University of Cambridge Repository with the identifier https://doi.org/10.17863/CAM.22990.
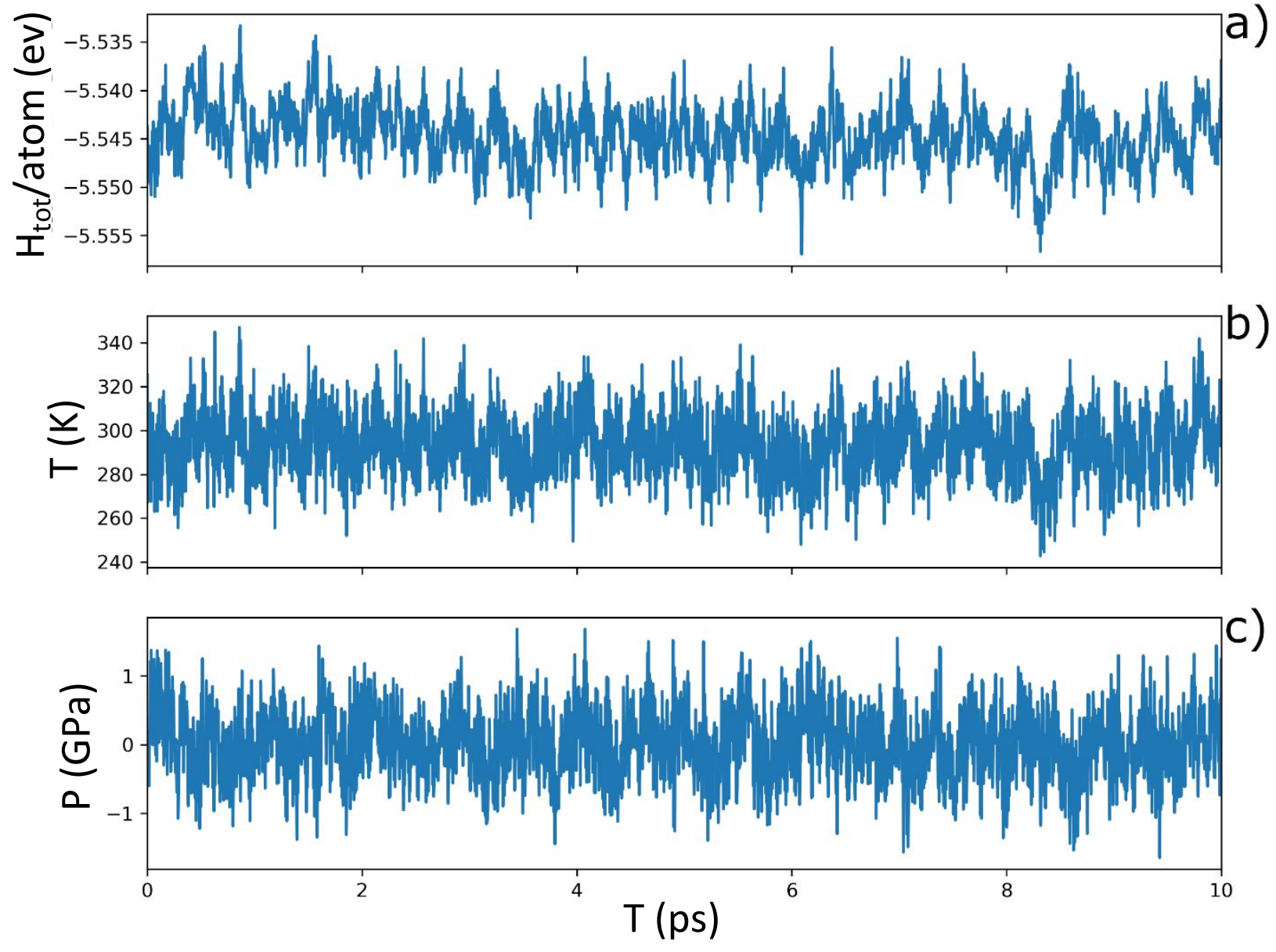

Figure S2 Time evolution of: a) the total enthalpy per atom $\left(H_{\text {tot }} /\right.$ atom $)$; b) temperature $(T)$ and c): pressure $(P)$ calculated from the initial $10 \mathrm{ps}$ of AIMD thermodynamic equilibration at $300 \mathrm{~K}$. 


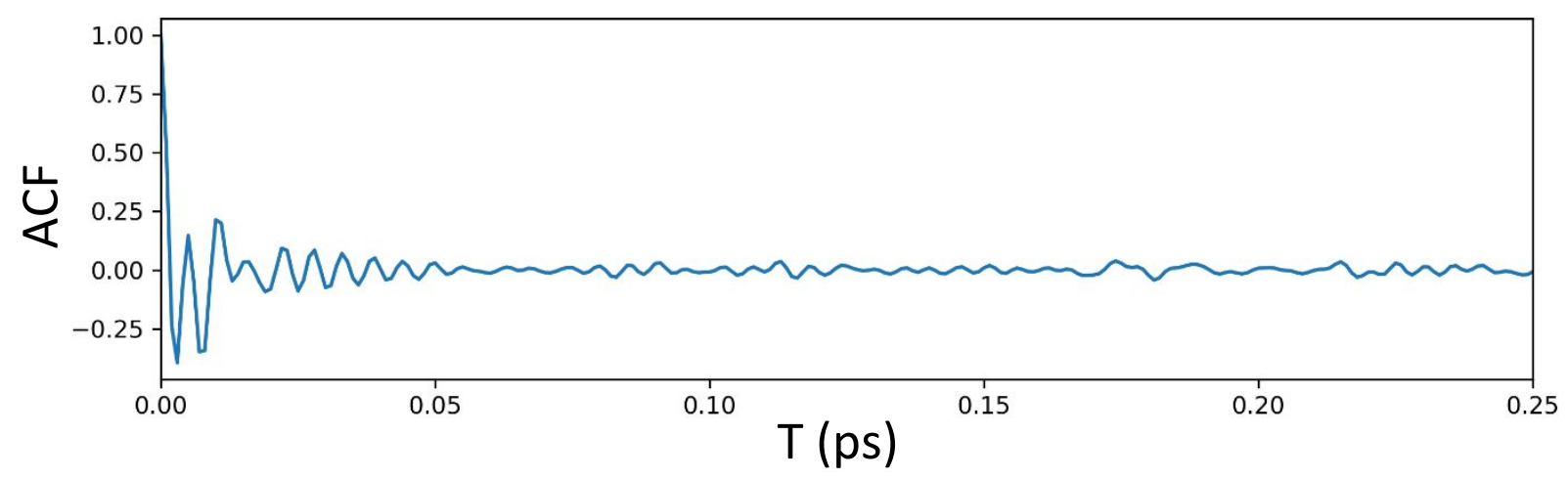

Figure S3 Time variation of the temperature auto-correlation function calculated from the initial $10 \mathrm{ps}$ of AIMD thermodynamic equilibration at $300 \mathrm{~K}$.

The simulation procedure was chosen to lie between the accuracy of ab initio methods and the capability of their classical counterpart to simulate models of much greater size and longer time scales. Initial-test AIMD runs revealed that, in order to perform the entire quench run with an $a b$ initio code exclusively on the HPC unit used in this study ( 2 x 12 core Intel Broadwell, 128GB RAM per node), one would have to obtain an equivalent of 2000h of continuous MD trajectory. Once the quench is done, MD runs at each studied temperature can be performed in parallel, where runs for the lowest studied temperature (longest runs) require around $240 \mathrm{~h}$ of continuous trajectory. Since the full $a b$ initio quench computation time and cost exceeded the project means, a force-field-based protocol was used for this part of the calculation. 


\section{Comparison between classical and $a b$ initio models}

In order to support our choice of the used ab initio model over a more computationally efficient classical approach, we have performed a detailed comparison of those two, with additional exploration of larger ensembles calculated within the latter approach. Overall, we have selected a 12-molecule (312-atom) ensemble - similar to that used for the ab initio approach and called here $1 \times 1 \times 1$, and a 96 -molecule (2462-atom) ensemble called $2 \times 2 \times 2$, and a 324-molecule (8424-atom) ensemble called $3 \times 3 \times 3$. Figure S4 shows oxygen-oxygen radial distribution functions calculated at $400 \mathrm{~K}$ for three classical ensemble sizes and one DFT ensemble. It is evident that patterns for all three classical data sets are of striking similarity, with only $1 \times 1 \times 1$ showing some differences compared to the others, most visibly for distances greater than $4 \AA$. A much greater difference is observed between the DFT pattern and any of the classical ones. The first peak, at around $2.75 \AA$, is visibly shifted towards lower values in the case of the DFT pattern compared to those of the FF origin. This reflects a major discrepancy in the potential-energy surface of the two models, indicating that the FF approach fails in reproducing the delicate interplay of forces governing the

dynamical properties investigated in this work. Additionally, two minor peaks, at around $3.7 \AA$ and $4.3 \AA$, are displaced in the FF patterns compared to the DFT one, and are much less visible. 


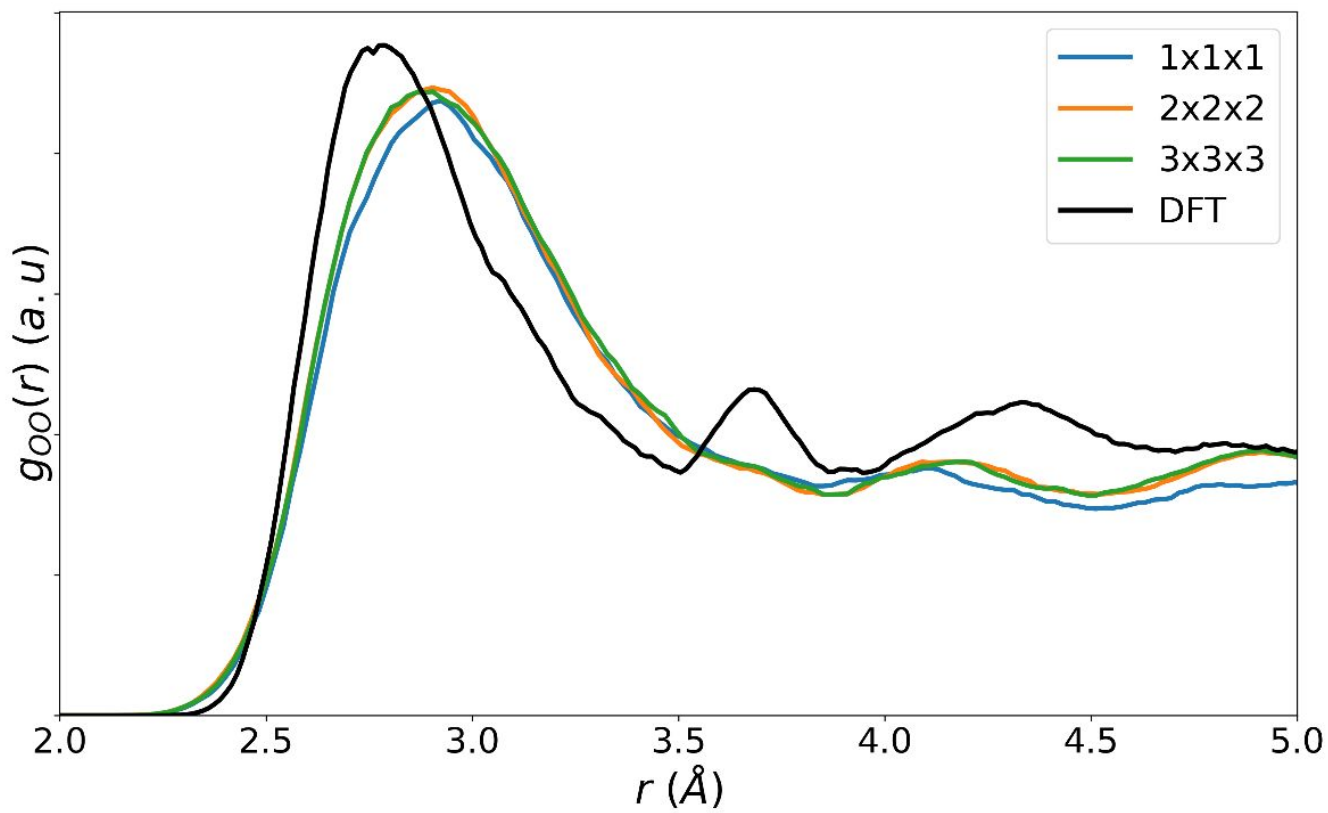

Figure S4 Radial distribution function of O-O pairs calculated at $400 \mathrm{~K}$ for three classical ensemble sizes (blue, orange and green) and one DFT ensemble.

To understand the observed difference between the presented patterns, the inter- and intramolecular oxygen-oxygen RDF patterns were calculated, based on the DFT data, and the results are presented in Figure S5. It is clear that the mentioned minor peaks, at around $3.7 \AA$ and $4.3 \AA$, are present only for the intramolecular pattern. This suggests that FF-based calculations do not properly reproduce the intramolecular geometry, despite the size of the ensemble. 


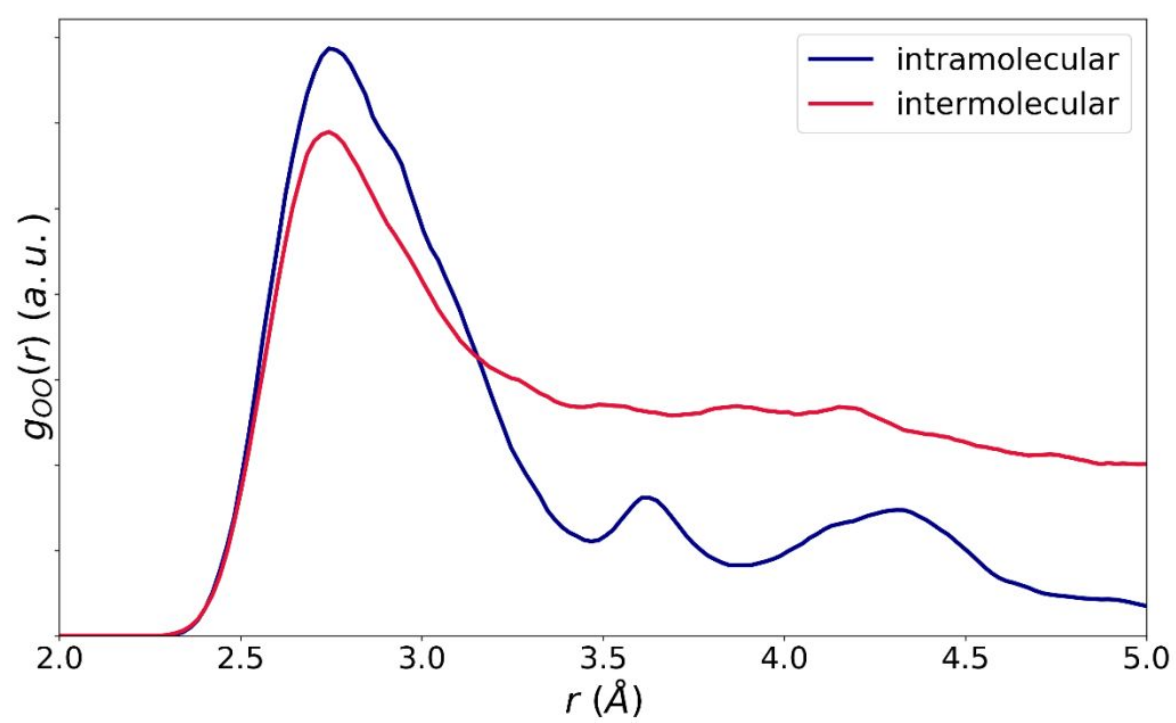

Figure S5 Inter- (red) and intra- (blue) molecular radial distribution function of O-O pairs calculated at $400 \mathrm{~K}$ for the DFT ensemble.

To further investigate differences between FF and DFT data, we have compared appropriate hydrogen-hydrogen radial distribution patterns for two hydrogen-ion sets: those with a rearrangement indicator $\sqrt{R_{r i}}>r_{\min }$ (considered as rearranging ions) and for a random subset of $\mathrm{H}$ ions. Figure S6 shows such a comparison for $400 \mathrm{~K}$ simulation data, respectively. In the case of the DFT model, the RDF patterns for the random set of $\mathrm{H}$ ions differs visibly from that for the set of "rearranging" ions - the first peak of the latter is much higher and of greater area than for the random set and suggests clustering of "rearranging" ions. For the FF-based RDF patterns, this difference is not observed. For the temperature $160 \mathrm{~K}$, as shown in Figure S7, the difference between random and "rearranging" sets of the FF model is more visible, but this change is even greater for the DFT model. This suggests that there is no evidence supporting the existence of dynamical heterogeneity in the classical model for the liquid phase, and only minor evidence for the solid state. 


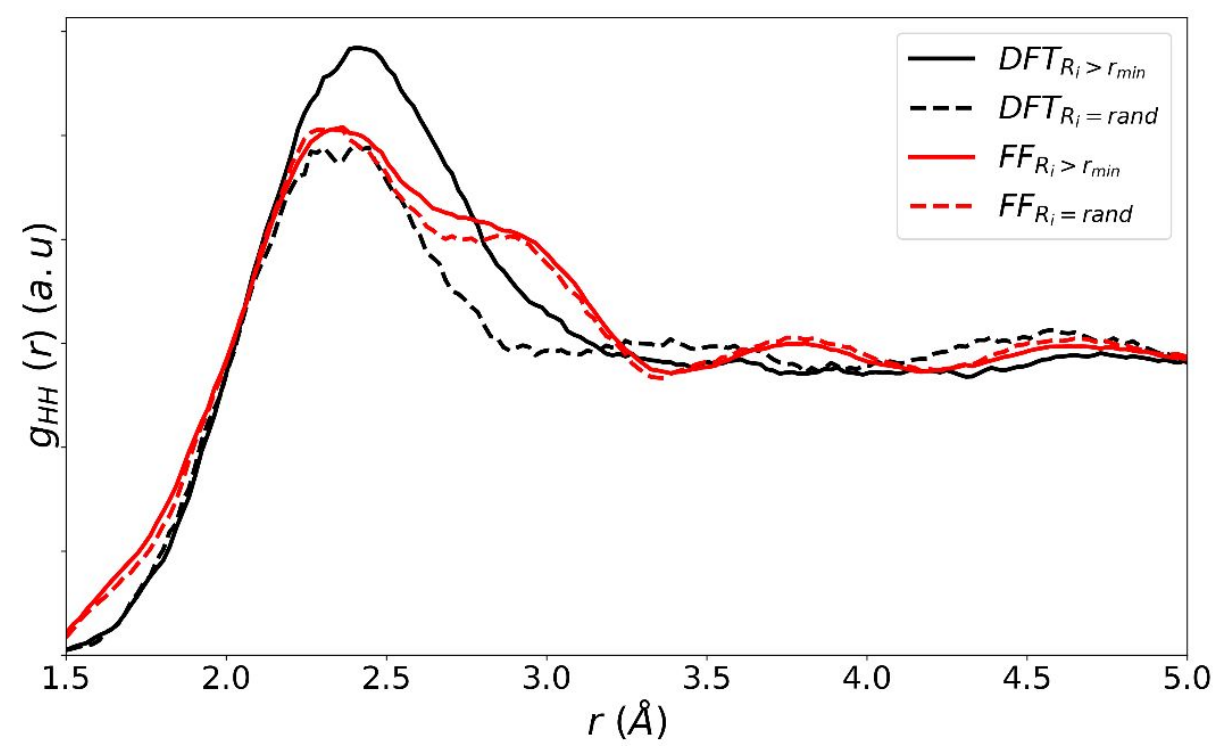

Figure S6 Radial distribution function (DFT - black, FF - red) of H-H pairs for "rearranging" ions (solid line) and a random set of hydrogen ions (dashed line). Data from $400 \mathrm{~K}$ simulations.

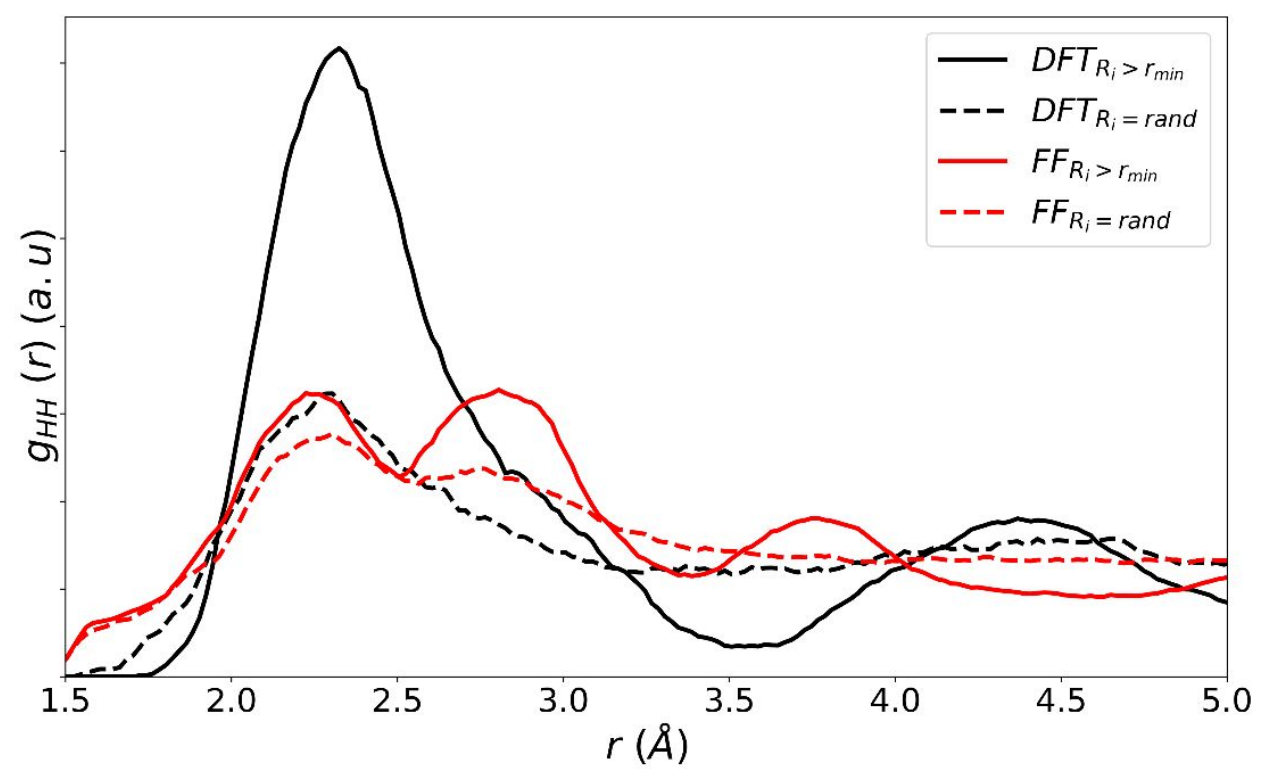

Figure S7 Radial distribution function (DFT - black, FF - red) of H-H pairs for "rearranging" ions (solid line) and a random set of hydrogen ions (dashed line). Data from $160 \mathrm{~K}$ simulations. S-9 
In summary, there are no major structural differences between all studied FF ensembles, but there are major structural and dynamical differences between DFT and FF models. This indicates that the FF approach fails in reproducing the delicate interplay of forces governing the structural dynamical properties investigated in this work. This fact justifies the need for FF simulations to be treated exclusively as the source of ionic coordinates that are later used as a starting point for DFT calculations. The size of the simulated ensemble of 312 atoms was chosen as the largest set of molecules for which the ab initio molecular dynamics could be carried out within reasonable cost. This size range is also widely accepted in the field where DFT studies on atomic and molecular glasses have been performed ${ }^{15,16}$.

\section{Validation of the ab initio model - experimental results}

In order to validate the DFT model, obtained radial distribution data were checked against the experimental results of X-ray diffraction. These were obtained by high-resolution X-ray PDF measurements using the I15-1 beam-line of the Diamond facility with a beam wavelength of $0.16169 \AA$. Figure S8 shows the radial distribution function from experiment and for the DFT model at $200 \mathrm{~K}$. It is clear that the characteristic features of the experimental pattern are well reproduced in the DFT model. The first O-O peak, at $2.8 \AA$, present in the ab initio pattern matches well, in both shape and the position, that observed in the X-ray data. This confirms that the density obtained for the DFT model stays in good agreement with the presented experimental data. 


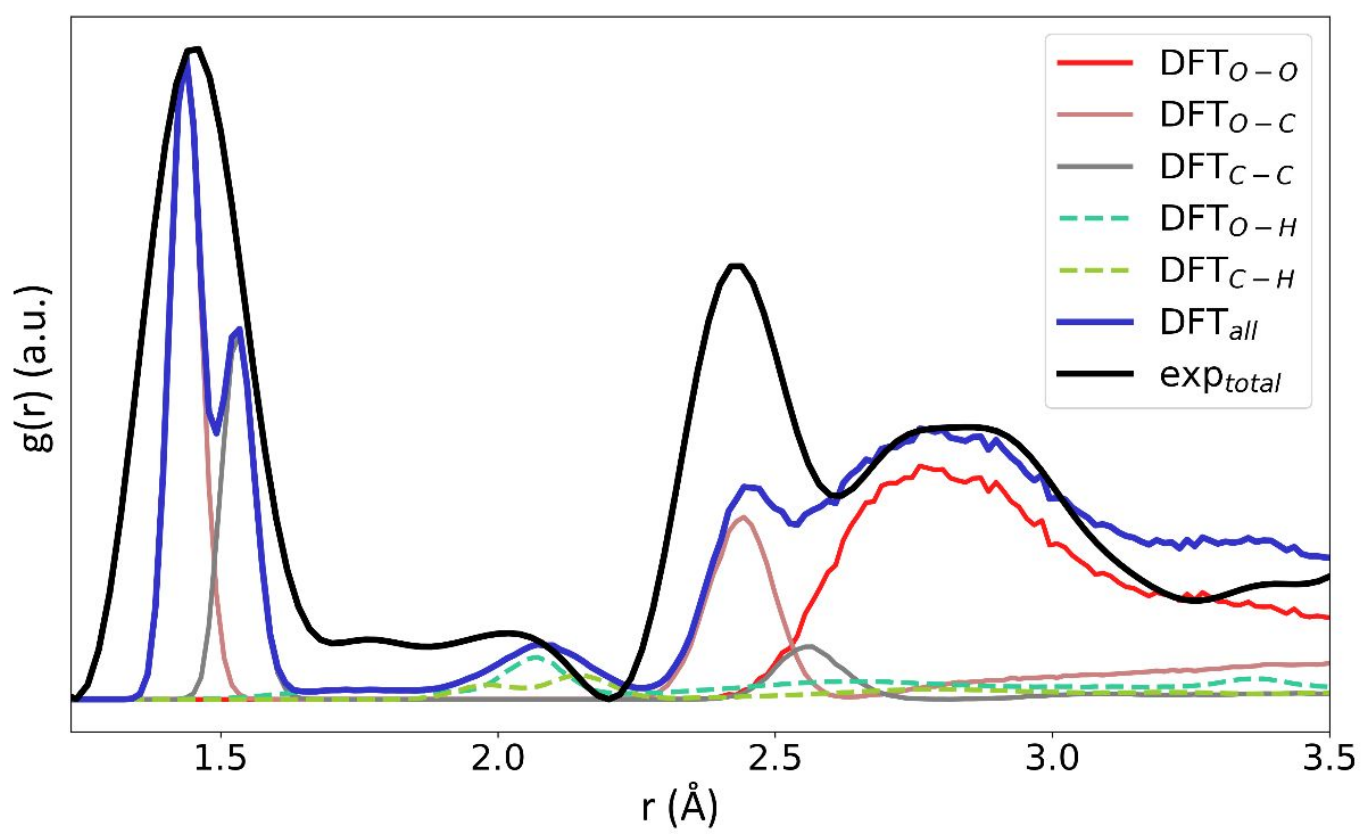

Figure S8 Radial distribution function of experimental (black - total) and DFT (O-O and O-C red and blue respectively) data at $200 \mathrm{~K}$.

\section{VDOS temperature dependence}

Hydrogen-atom mobility was initially investigated by means of the atom-specific vibrational density of states, $V D O S_{a}$, as a function of frequency, $f$, defined as:

$$
\operatorname{VDOS}_{a}(f)=\frac{1}{3 N_{a} T k_{B}} \int_{-\infty}^{\infty} \sum_{i=1}^{N_{a}}\left\langle V C F_{i}(t)\right\rangle e^{2 \pi f t} d t
$$

where $N_{a}$ is the number of $a$-type atoms, $T$ is the temperature, $k_{B}$ is the Boltzmann factor and $V C F_{i}$ $(t)=\left\langle\mathbf{v}_{\mathrm{i}}(0) \cdot \mathbf{v}_{\mathrm{i}}(t)\right\rangle$ is the velocity correlation function of atom $i$. Figure S9 (black curve) shows the $\operatorname{VDOS}_{a}$ pattern $(a=\mathrm{H})$ for glassy d-sorbitol, calculated for trajectories of hydrogen atoms in hydroxyl groups at $240 \mathrm{~K}$. The pattern is characterized by three well-pronounced peaks, at around 20, 41 and $108 \mathrm{THz}$ (or 668,1368 and $3602 \mathrm{~cm}^{-1}$, respectively). It suggests that the majority of the 
hydrogen-atom movement can be decomposed into three independent modes: two-body (H-O stretch), three-body (C-O-H bend) and four-body (C-C-O-H torsion). These interactions were analyzed by calculating vibrational density of states patterns, $V D O S_{r}, V D O S_{\alpha}$ and $V D O S_{\varphi}$, respectively, according to equation (1), with velocity autocorrelation functions respectively defined as: $\left\langle\dot{\mathbf{r}}_{\mathrm{i}}(0) \cdot \dot{\mathbf{r}}_{\mathrm{i}}(t)\right\rangle$, where $\dot{\mathbf{r}}_{\mathrm{i}}$ is the $O_{i}-H_{i}$ distance time derivative; $\left\langle\dot{\alpha}_{\mathrm{i}}(0) \cdot \dot{\alpha}_{\mathrm{i}}(t)\right\rangle$, where $\dot{\alpha}_{\mathrm{i}}$ is the $\mathrm{C}_{\mathrm{i}}-\mathrm{O}_{\mathrm{i}}-\mathrm{H}_{\mathrm{i}}$ bond-angle time derivative; and $\left\langle\dot{\varphi}_{\mathrm{i}}(0) \cdot \dot{\varphi}_{\mathrm{i}}(t)\right\rangle$, where $\dot{\varphi}_{\mathrm{i}}$ is the $\mathrm{C}_{\mathrm{j}}-\mathrm{C}_{\mathrm{i}}-\mathrm{O}_{\mathrm{i}}-\mathrm{H}_{\mathrm{i}}$ dihedralangle time derivative. Figure S9 shows $\operatorname{VDOS}_{r}$ (blue), $V D O S_{\alpha}$ (green) and $V D O S_{\varphi}$ (red) patterns calculated from $240 \mathrm{~K} \mathrm{MD}$ trajectories. It is worth noting that $V D O S_{H}, V D O S_{r}, V D O S_{\alpha}$ and $V D O S_{\varphi}$ are defined with different units so, for the sake of comparison, they have been scaled with frequency-independent coefficients, $C_{\mathrm{r}}, C_{\alpha}$ and $C_{\varphi}$, respectively, so that their linear combination, $V D O S_{\text {calc }}=C_{r} V D O S_{r}+C_{\alpha} V D O S_{\alpha}+C_{\varphi} V D O S_{\varphi}$, gives the best approximation to $V D O S_{H}$. The peaks in the vibrational density of states for individual motion types match well the characteristic peaks of the $V D O S_{H}$ pattern, indicating that the movement of hydroxyl-group hydrogen atoms can indeed be simplified to three independent atomic-movement patterns. 


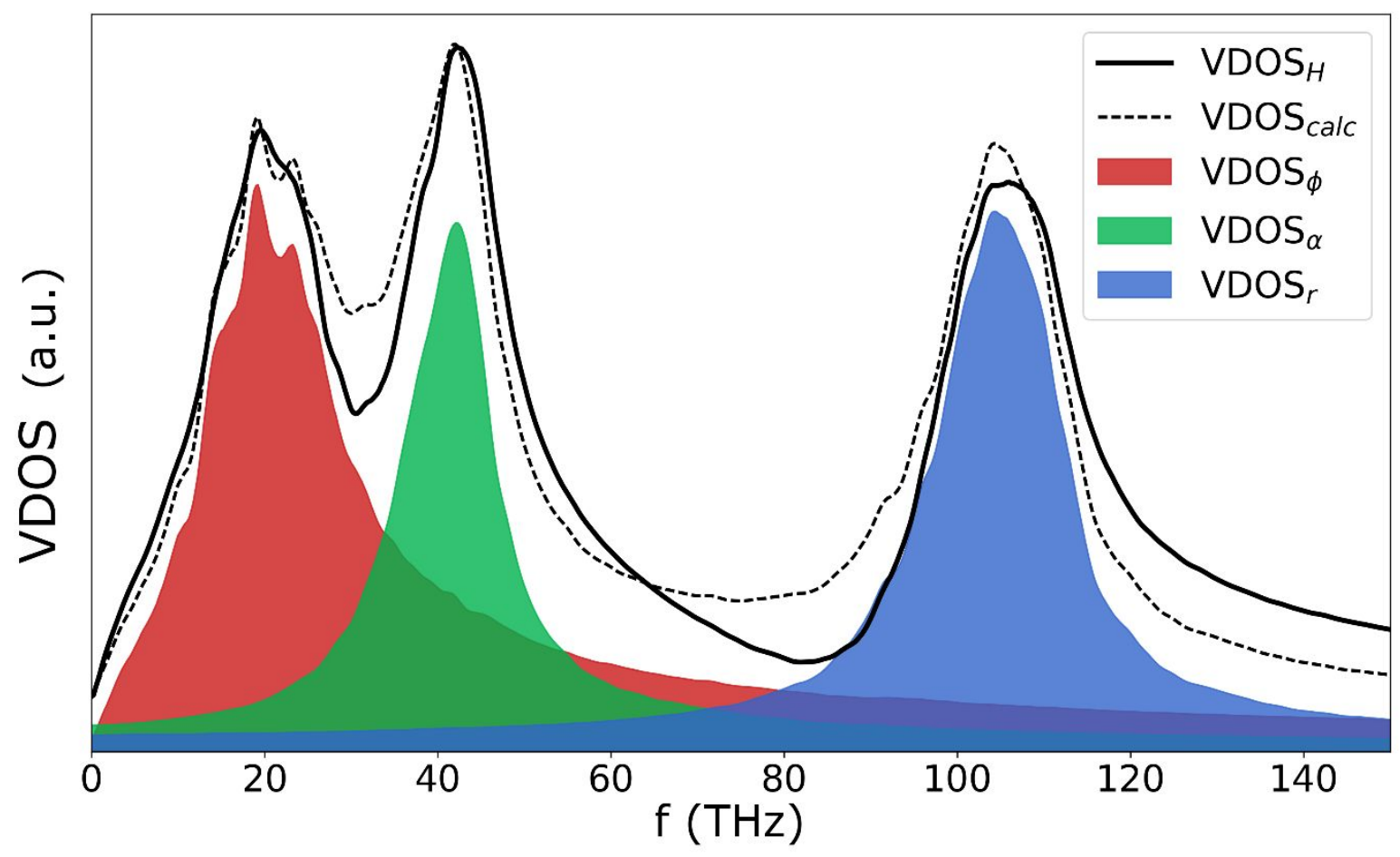

Figure S9 Partial VDOS calculated for: (black solid curve) hydroxyl-group hydrogen ions $\left(V_{D O S}\right)$; (red) $\mathrm{C}_{\mathrm{j}}-\mathrm{C}_{\mathrm{i}}-\mathrm{O}_{\mathrm{i}}-\mathrm{H}_{\mathrm{i}}$ dihedral torsion; (green) $\mathrm{C}_{\mathrm{i}}-\mathrm{O}_{\mathrm{i}}-\mathrm{H}_{\mathrm{i}}$ bend; (blue) $\mathrm{O}_{\mathrm{i}}-\mathrm{H}_{\mathrm{i}}$ stretch; (black dashed curve) linear combination, $V D O S_{\text {calc }}$ of $V D O S_{\phi}, V D O S_{\alpha}$ and $V D O S_{r}$, calculated as the best fit to $V D O S_{H}$.

Of the three characteristic H-related peaks in the VDOS, two exhibit visible shifts with changing temperature (Figure S10a). The characteristic frequency of the $\mathrm{OH}$-stretch increases visibly with increasing temperature. Interestingly, the low-frequency peak at around $20 \mathrm{THz}$, associated with conformational motions of $\mathrm{C}_{\mathrm{j}}-\mathrm{C}_{\mathrm{i}}-\mathrm{O}_{\mathrm{i}}-\mathrm{H}_{\mathrm{i}}$ quartets, shifts towards lower frequencies with increasing temperature. A detailed analysis of the peak-shift temperature dependency was performed by calculating the modulus sum of peak shifts, $\Delta f_{V D O S}(T)=\left|f_{V D O S_{r}}(T)-f_{V D O S_{r}}\left(T_{0}\right)\right|+$ $\left|f_{\text {VDOS }_{\phi}}(T)-f_{\text {VDOS }_{\phi}}\left(T_{0}\right)\right|$, taking $T_{0}$ to be $80 \mathrm{~K}$. Figure S10b shows the temperature variation of $\Delta$ 
$f_{V D O S}$, revealing a markedly non-linear temperature dependency, with the behavior divided into three characteristic regions, separated by (experimental values of) $T_{\beta}$ and $T_{\alpha}$ (Figure $\mathrm{S} 10(\mathrm{~b})$ ).
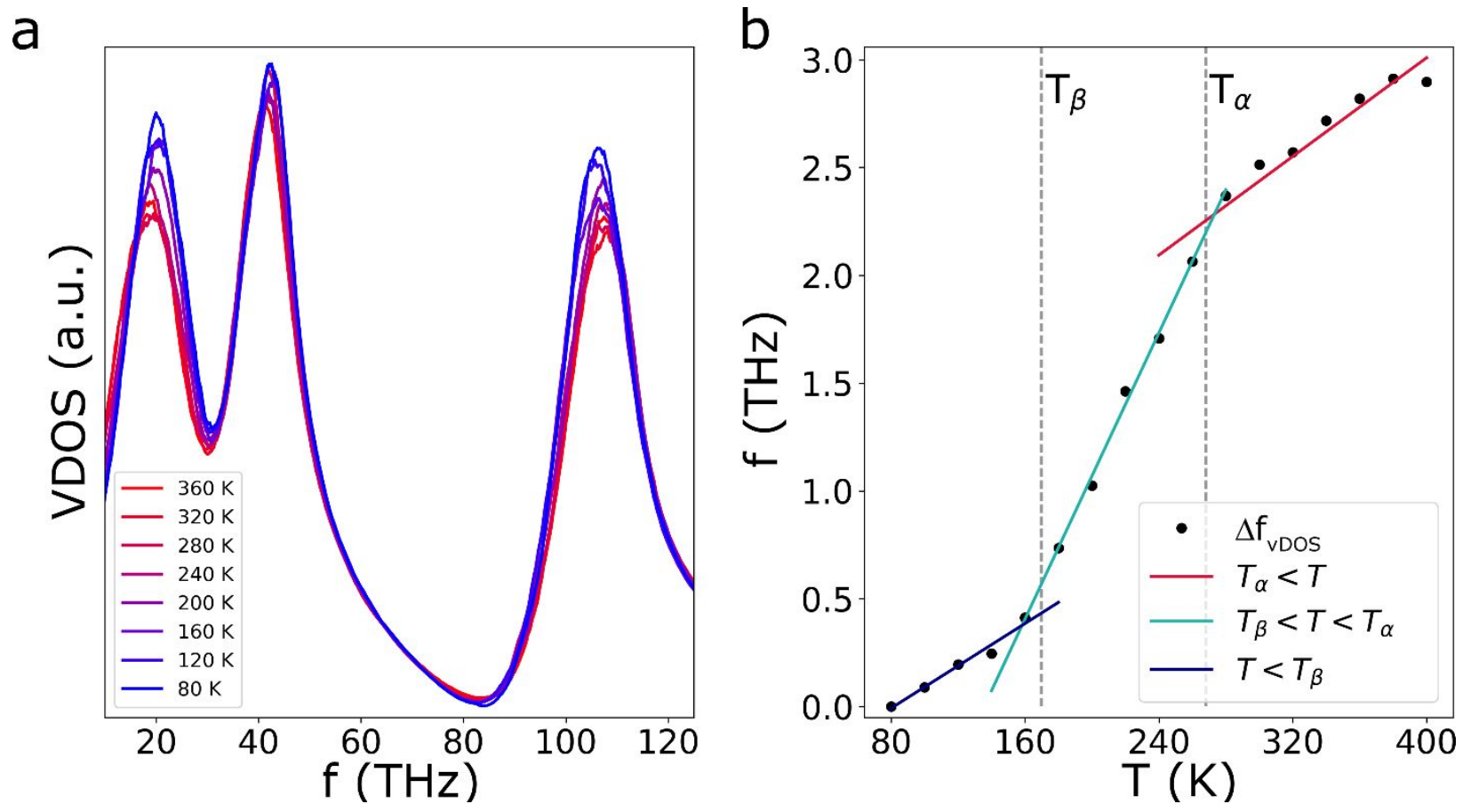

Figure S10 (a) Hydroxyl-group hydrogen-ion $\operatorname{VDOS}_{H}$, calculated at selected temperatures. (b) Temperature variation of the absolute sum of the frequency shifts, $\Delta f_{V D O S}$, for the peaks at $\sim 20$ and $\sim 110 \mathrm{THz}$. Blue, cyan and red colors represent linear approximations to $\Delta f_{V D O S}$ data in the temperature ranges $T<T_{\beta}, T_{\beta}<T<T_{\alpha}$ and $T_{\alpha}<T$, respectively, where $T_{\alpha}$ and $T_{\beta}$ are the experimental values of the corresponding glass transitions ${ }^{17}$. 


\section{Minimal dihedral- and spatial-rearrangement calculation}

To calculate the angle of rotation, one has to find the projection of an acceptor ion $O_{A 1}$ and a competing ion $O_{A 2}$ on the surface that is perpendicular to the dihedral-rotation axis $\left(C_{3^{-}} O_{D}\right.$ in Figure S11). The angle $\delta$ between the acceptor projected position $P_{l}$, donor oxide ion $O_{D}$, and competing-acceptor projected position $P_{2}$, is a good estimate for the dihedral rotation observed during hydrogen-bond rearrangement. A corresponding analysis shows that, for every studied temperature, there is a single peak present in the distribution for $\delta$, with a maximum around $54^{\circ}$ (see Figure S12a). This threshold value is chosen semi-arbitrarily, but tests show that deviations of $\pm 5^{0}$ from this value do not noticeably affect the results of the analysis. Similarly to the minimal dihedral-rearrangement angle, $\delta_{\min }$, a minimum spatial-relocation distance, $r_{m i n}$, between points $P_{1}$ and $P_{2}$ was calculated at various temperatures (Figure $\mathrm{S} 12 \mathrm{~b}$ ), with the distribution showing a single peak at around $0.86 \AA$. For further analysis, we assume that the minimal spatial-relocation distance associated with the dihedral motion therefore needs to be greater than $r_{\text {min }}=0.43 \AA$. 


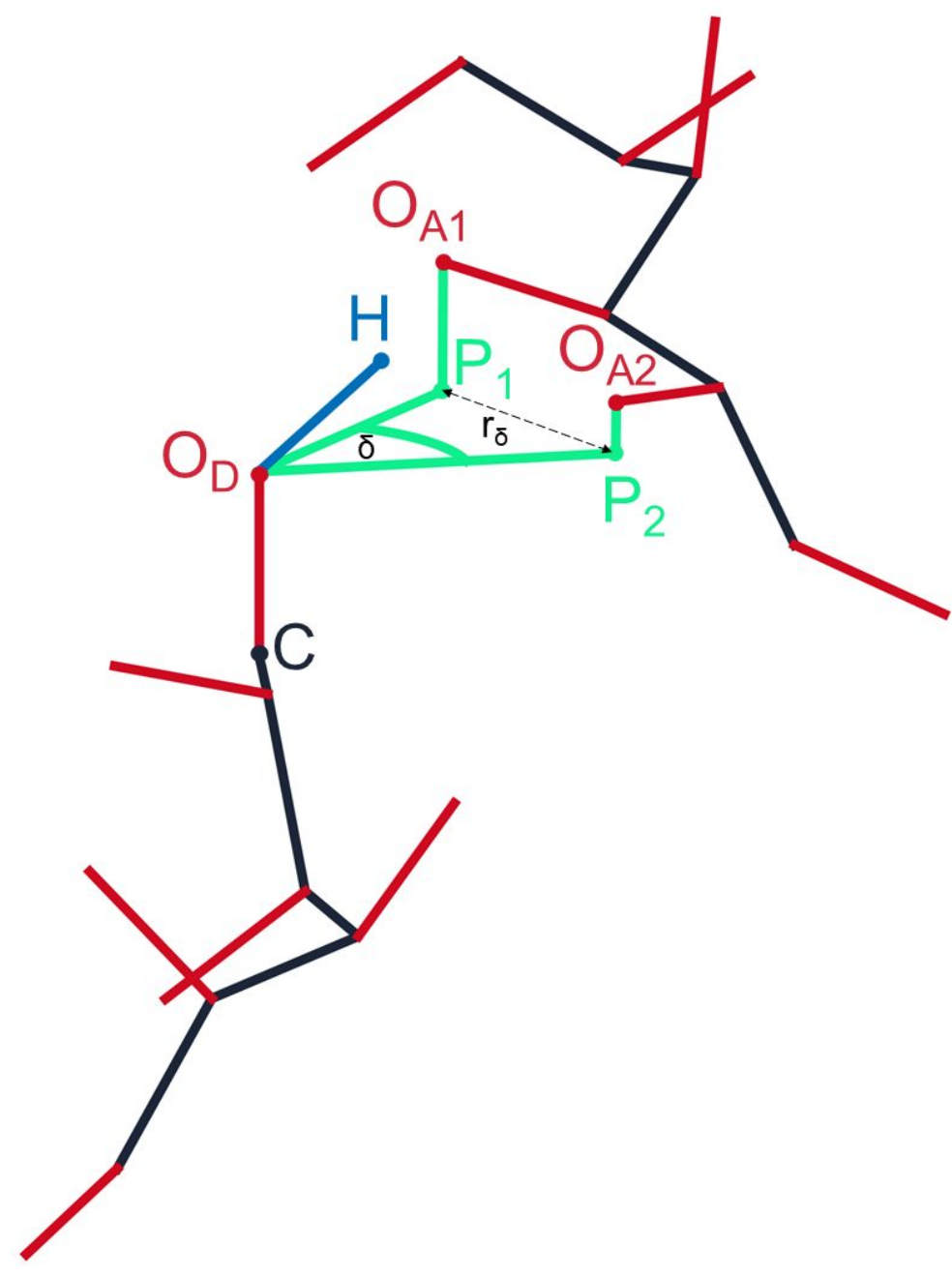

Figure S11 Schematic representation of two sorbitol molecules with a highlighted hydrogen ion, $\mathrm{H}$ (blue), during hydrogen-bond relocation between acceptor ions $\mathrm{O}_{\mathrm{A} 1}$ and $\mathrm{O}_{\mathrm{A} 2}$. The dihedral torsion angle observed during bond rearrangement is marked by $\delta$ and the spatial-relocation distance by $r_{\delta}$. 

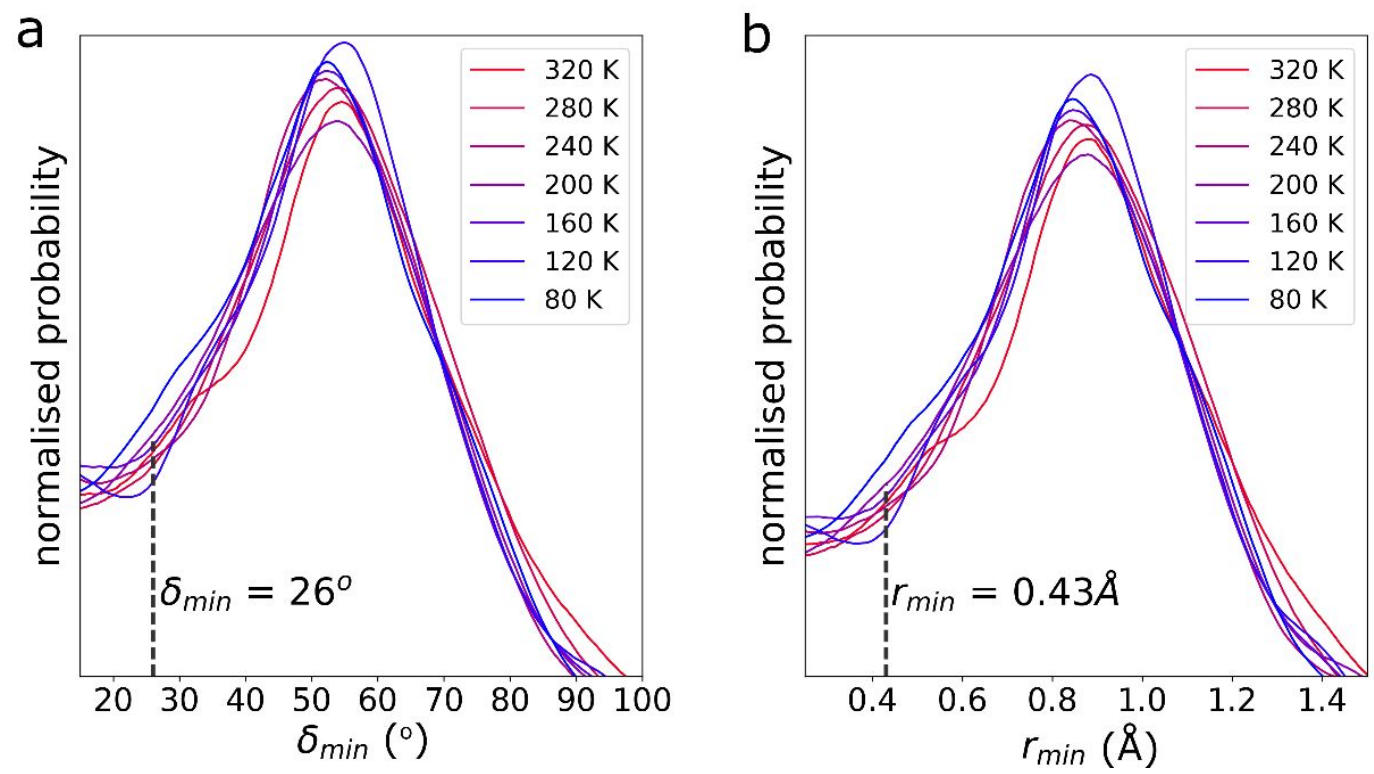

Figure S12 Probability distribution of: (a) dihedral angles; and (b) spatial distances between two oxide-ion acceptors participating in hydrogen-bond formation, calculated at different temperatures, as marked. $\delta_{\min }=26^{\circ}$ and $r_{\min }=0.43 \AA$ are chosen as threshold values, above which dihedral-angle and spatial relocations are considered to be large enough so that hydrogenbond breakage and reformation might be observed. 


\section{Bond-reformation temporal analysis}

Additionally, the time cross-correlation function was calculated for different pairs of atoms (all results given in Table $\mathrm{S} 1$ ). This shows a very high correlation between $\mathrm{O}_{\mathrm{i}} \mathrm{H}_{\mathrm{i}}, \mathrm{C}_{\mathrm{i}} \mathrm{O}_{\mathrm{i}}$ and $\mathrm{C}_{\mathrm{i}} \mathrm{C}_{1 \text { st.n.n. }}$ doublets, reaching values of $0.59,0.61$ and 0.61 , respectively. Interestingly, fairly high rearrangement correlations, with values of 0.20 and 0.15 , were observed for pairs of hydrogen ions, $\mathrm{H}_{i} \mathrm{H}_{1 \text { st.n.n }}$, separated by $\mathrm{C}-\mathrm{O}-\mathrm{O}-\mathrm{C}$ atoms, and $\mathrm{H}_{\mathrm{i}} \mathrm{H}_{2 \text { nd.n.n. }}$ separated by $\mathrm{O}-\mathrm{C}-\mathrm{C}-\mathrm{C}-\mathrm{O}$ atoms, respectively (see Figure S11). Furthermore, a correlation of 0.2 was observed for the $\mathrm{H}_{\mathrm{i}} \mathrm{H}_{\mathrm{h} \text {-bond }}$ atoms of a donor/acceptor hydroxyl group of a single hydrogen bond. Further study of rearrangement correlations reveals a delay of the first peak present for all investigated patterns of 5.6 fs and 12 fs for $\mathrm{H}_{i} \mathrm{H}_{1 \text { st.n.n }}$ and $\mathrm{H}_{\mathrm{i}} \mathrm{H}_{\text {h-bond }}$, respectively.

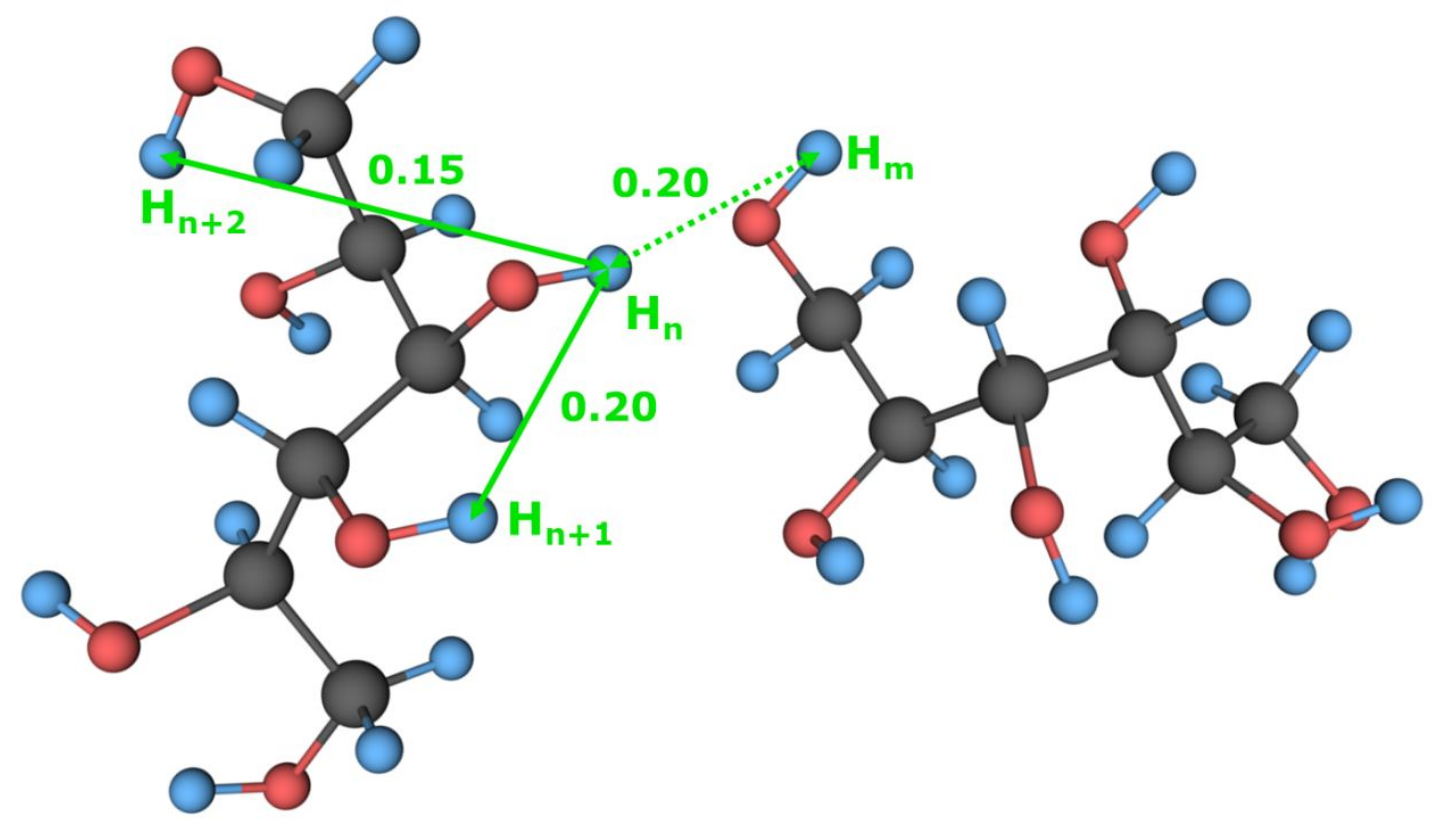

Figure S13 Model representation of the rearrangement correlations observed for different pairs of ions, according to the data presented in Table S1. Blue, red and black spheres correspond to hydrogen, oxygen and carbon ions, respectively, of the sorbitol molecule. 
Table S1 Correlations calculated for different pairs of atoms: $A_{i} B_{i}$ denotes atoms in the same $\mathrm{COH}$ triplet; $\mathrm{A}_{\mathrm{i}} \mathrm{A}_{(1 \text { st.n.n.) }}$ denotes an atom and its first nearest neighbor; $\mathrm{A}_{\mathrm{i}} \mathrm{A}_{(2 \text { nd.n.n.) }}$ denotes an atom and its second nearest neighbor; $\mathrm{A}_{\mathrm{i}} \mathrm{A}_{\text {(h-bond) }}$ denotes a donor hydrogen atom and a hydrogen atom of the acceptor hydroxyl group for the same hydrogen bond.

\begin{tabular}{ll} 
atom pair & cross-correlation \\
\hline $\mathrm{O}_{\mathrm{i}} \mathrm{H}_{\mathrm{i}}$ & $0.59(0.03)$ \\
$\mathrm{C}_{\mathrm{i}} \mathrm{O}_{\mathrm{i}}$ & $0.61(0.01)$ \\
$\mathrm{C}_{\mathrm{i}} \mathrm{O}_{\mathrm{i}}$ & $0.39(0.02)$ \\
$\mathrm{C}_{\mathrm{i}} \mathrm{C}_{\text {1st.n.n. }}$ & $0.61(0.02)$ \\
$\mathrm{C}_{\mathrm{i}} \mathrm{C}_{2 \text { nd.n.n. }}$ & $0.29(0.01)$ \\
$\mathrm{H}_{\mathrm{i}} \mathrm{H}_{1 \text { st.n.n. }}$ & $0.20(0.01)$ \\
$\mathrm{H}_{\mathrm{i}} \mathrm{H}_{2 \text { nd.n.n. }}$ & $0.15(0.01)$ \\
$\mathrm{H}_{\mathrm{i}} \mathrm{H}_{\text {h-bond }}$ & $0.20(0.11)$
\end{tabular}




\section{Additional results}

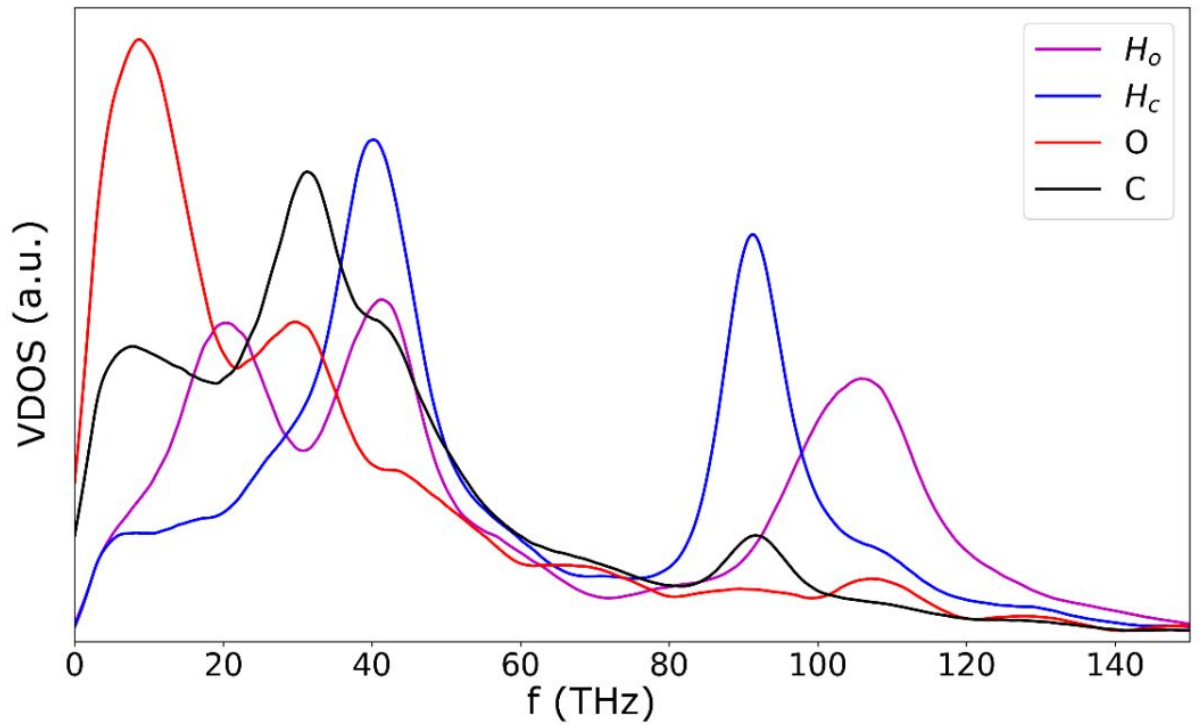

Figure S14 Vibrational density of states calculated for: (purple) hydroxyl-group hydrogens ions;

(blue) carbon-ion-bonded hydrogen ions; (red) oxide ions; and (black) carbon ions.
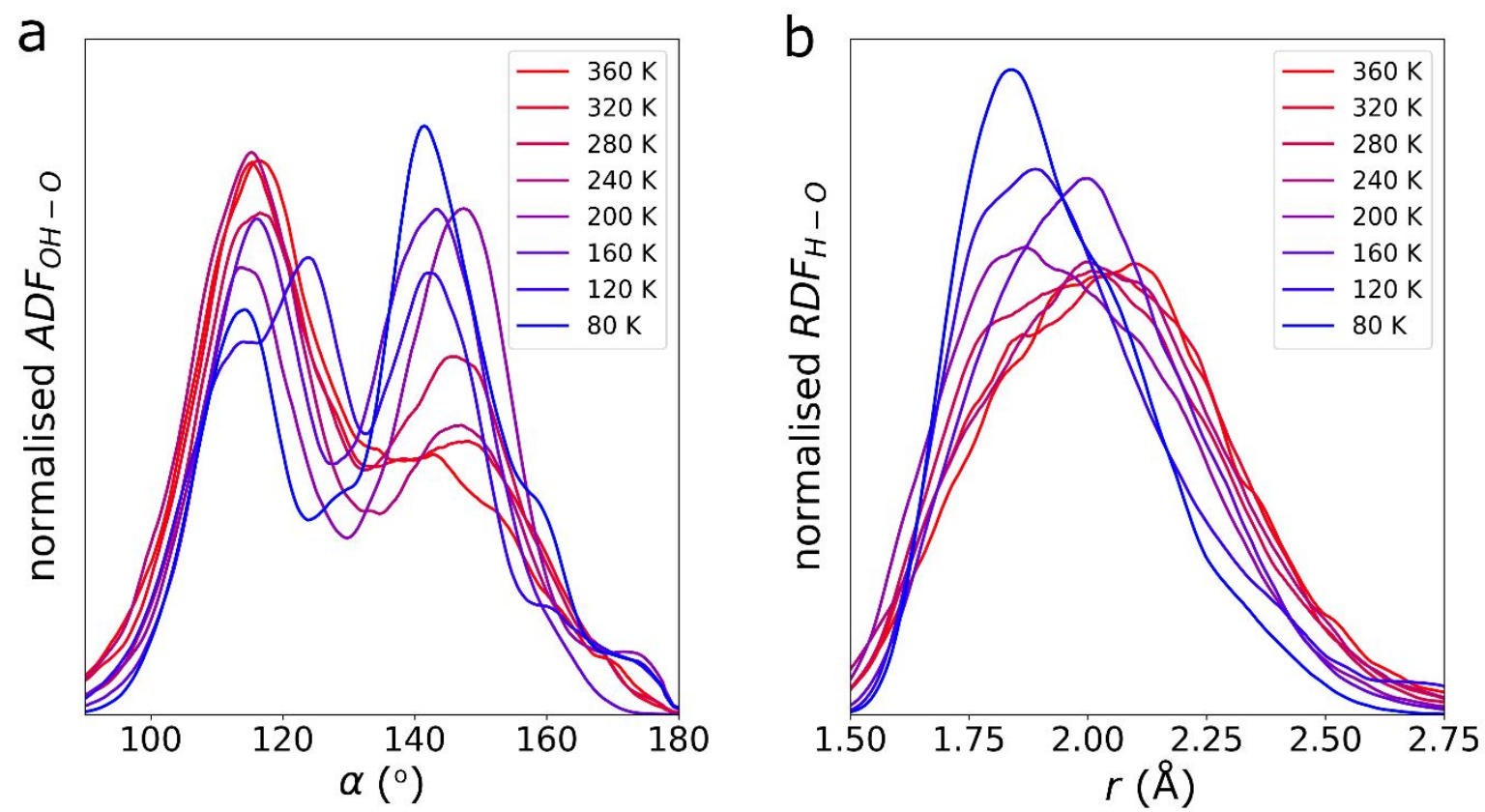

Figure S15 (a) $\mathrm{ADF}_{\mathrm{OH}-\mathrm{O}}$ and (b) $\mathrm{RDF}_{\mathrm{H}-\mathrm{O}}$ patterns calculated for intramolecular hydrogen bonds at selected temperatures. 

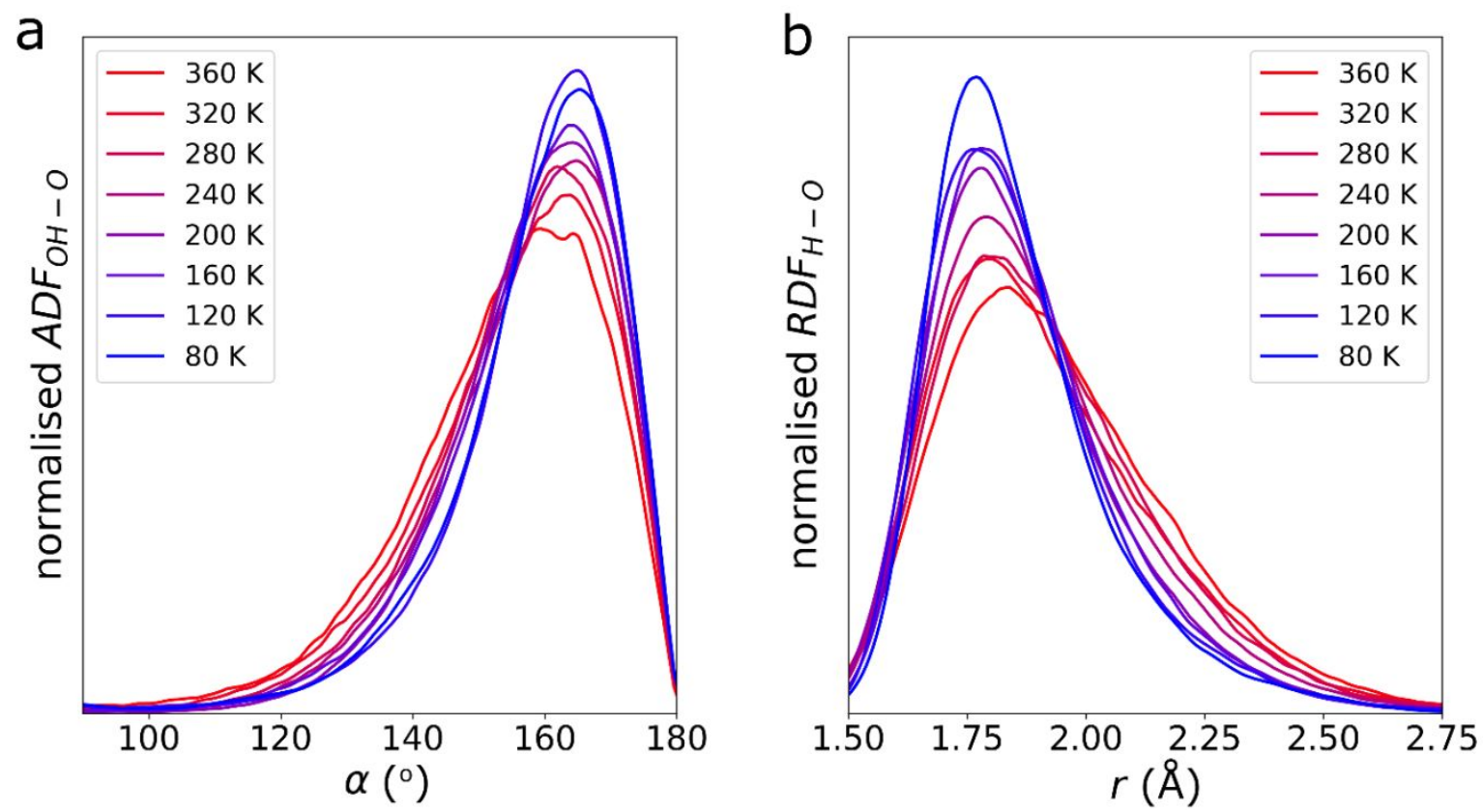

Figure S16 Normalised (a) $\mathrm{ADF}_{\mathrm{OH}-\mathrm{O}}$ and (b) $\mathrm{RDF}_{\mathrm{H}-\mathrm{O}}$ patterns calculated for intermolecular hydrogen bonds at selected temperatures.

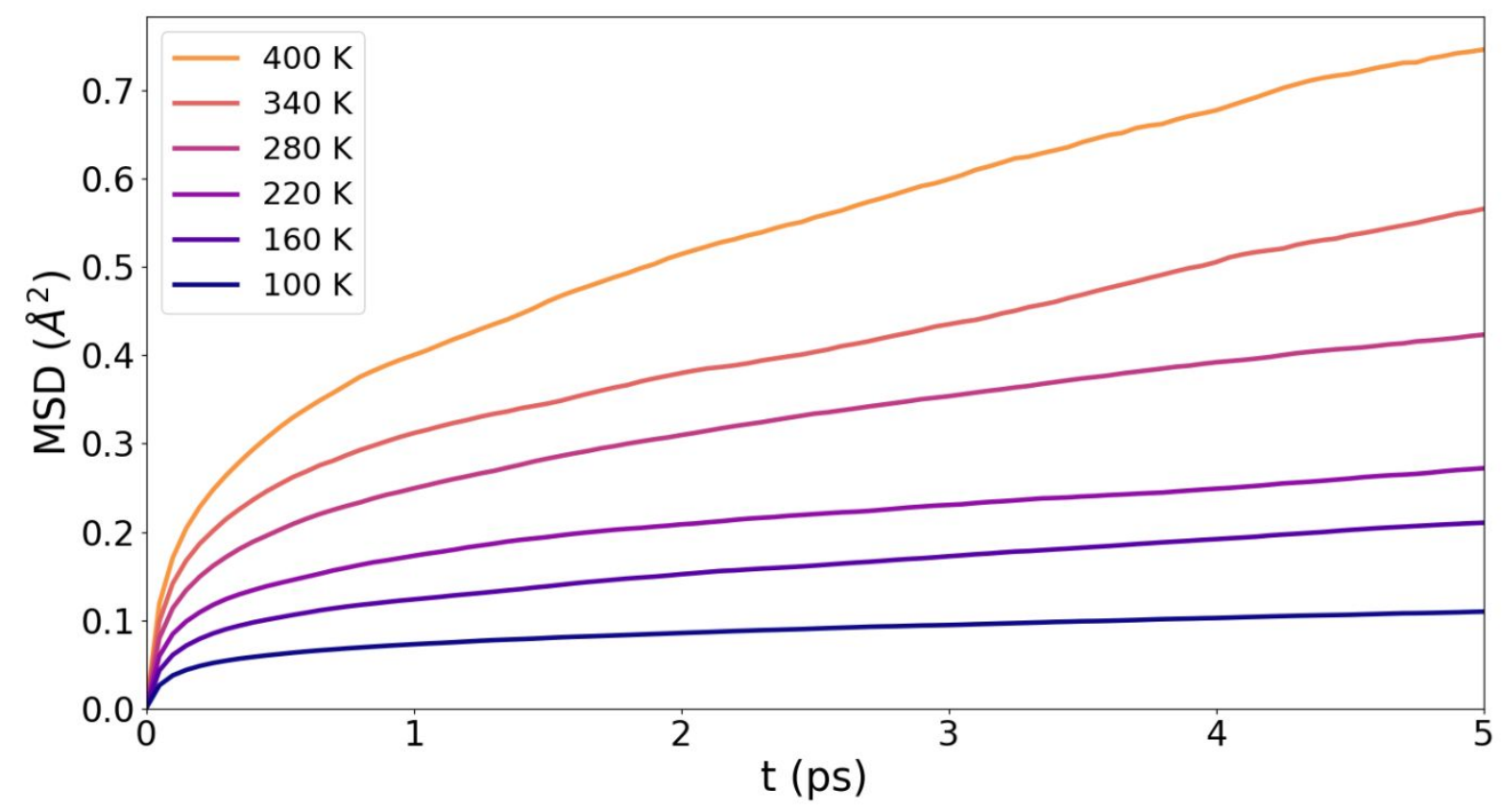

Figure S17 Time evolution of the mean-square displacement of hydrogen ions calculated for dsorbitol. 

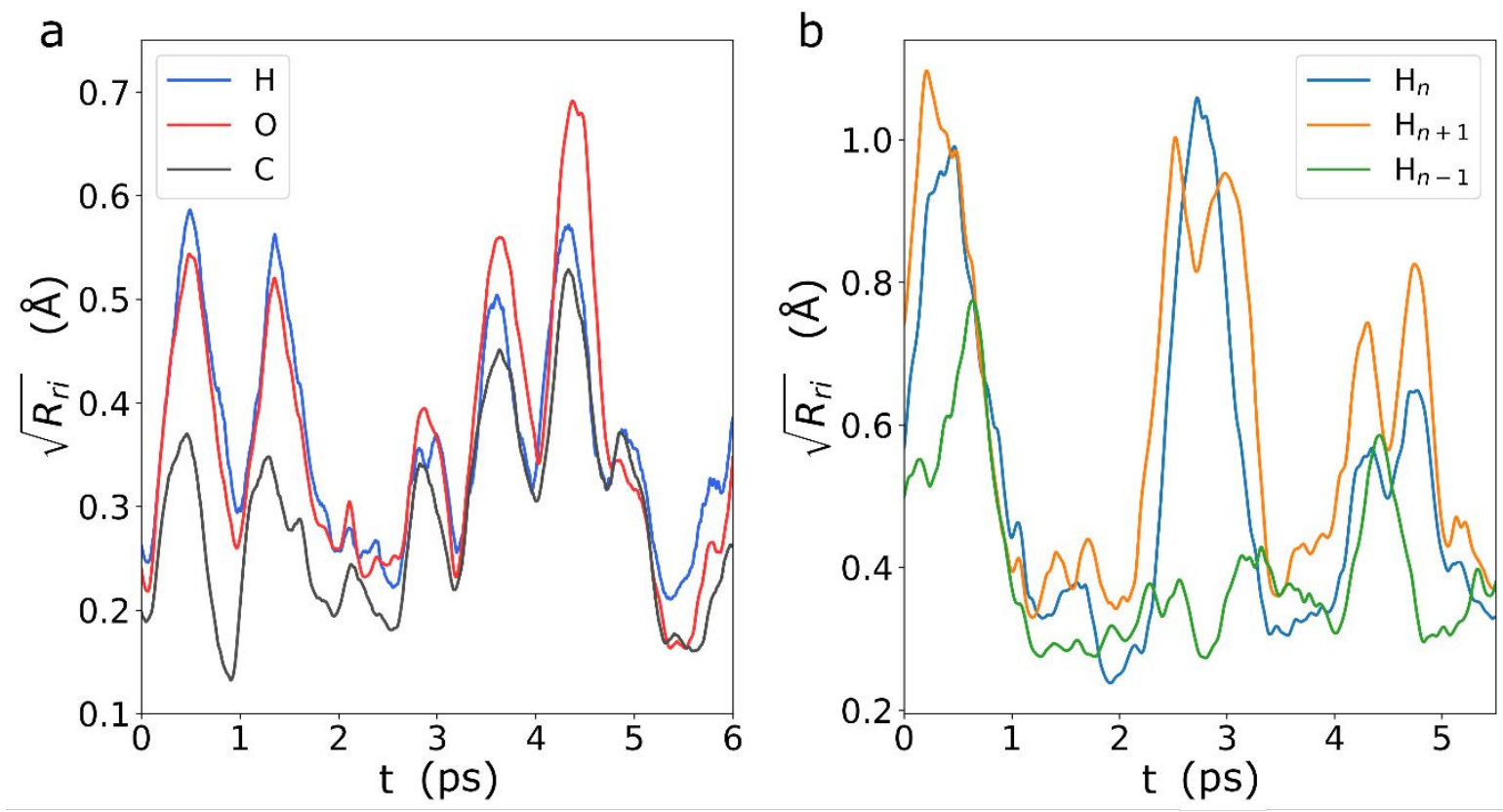

Figure S18 Time evolution of the square root of the spatial-rearrangement indicator, $\sqrt{R_{r i}}$, calculated for: (a) three neighboring atoms, $\mathrm{H}$ (blue), $\mathrm{O}$ (red) and C (black); (b) three neighboring hydrogen ions in the same molecule. The data correspond to a $300 \mathrm{~K}$ MD run. 


\section{References}

(1) Hammer, B.; Hansen, L. B.; Nørskov, J. K. Improved Adsorption Energetics Within DensityFunctional Theory Using Revised Perdew-Burke-Ernzerhof Functionals. Phys. Rev. B 1999, 59, 7413-7421.

(2) Kresse, G.; Joubert, D., From Ultrasoft Pseudopotentials to the Projector Augmented-Wave Method. Phys Rev B 1999, 59, 1758-1775.

(3) Parrinello M.; Rahman A. Crystal Structure and Pair Potentials: A Molecular-Dynamics Study. Phys. Rev. Lett. 1980, 45, 1196.

(4) Rahman A.; Parrinello M. Polymorphic Transitions in Single Crystals: a New Molecular Dynamics Method. J. Appl. Phys. 1981, 52, 7182.

(5) Monkhorst, H. J.; Pack, J. D. Special Points for Brillouin-Zone Integrations. Phys Rev B 1976, $13,5188-5192$.

(6) Grimme, S.; Antony, J.; Ehrlich, S.; Krieg, S. A Consistent and Accurate Ab Initio Parametrization of Density Functional Dispersion Correction (DFT-D) For the 94 Elements H-Pu. J. Chem. Phys. 2010, 132, 154104.

(7) Grimme, S., Ehrlich, S. \& Goerigk, L., Effect of the Damping Function in Dispersion Corrected Density Functional Theory. J. Comp. Chem. 2011, 32, 1456-1465.

(8) Limmer, D. T. The Lengtha and Time Scales of Water's Glass Transitions. J. Chem. Phys. 2014, 140, 214509.

(9) Zheng, G. P.A Density Functional Theory Study on the Deformation Behaviors of Fe-Si-B Metallic Glasses. Int. J. Mol. Sci., 2012, 13, 10401-10409. 
(10) Buchholz, J.; Paul, W.; Varnik, F.; Binder, K. Cooling Rate Dependence of the Glass Transition Temperature of Polymer Melts: Molecular Dynamics Study. J. Chem. Phys. 2002, 117, 7364.

(11) Li, X.; Song, W.; Yang, K.; M.Anoop Krishnan A.; Wang, B.; Smedskjaer, M. M.; Mauro, J. C.; Sant, G.; Balonis, M.; Bauchy, M. Cooling Rate Effects in Sodium Silicate Glasses. J. Chem. Phys., 2017, 147, 074501.

(12) Roux, S.; Jund, P.; Influence of the Cooling Rate on the Glass Transition Temperature and the Structural Properties of Glassy Ges 2 : An Ab Initio Molecular Dynamics Study. J. Phys. Condens. Matter 2007, 19, 19.

(13) Caprion, D.; Schober, H. R.; Influence Of The Quench Rate and the Pressure on the Glass Transition Temperature in Selenium. J. Chem. Phys. 2002, 6, 117.

(14) Wang, J.; Hodgson, P. D.; Zhang, J.; Yan, W.; Yang, C. Effects of Quenching Rate on Amorphous Structures of $\mathrm{Cu}_{46} \mathrm{Zr}_{54}$ Metallic Glass. J. Mater. Process. Technol. 2009, 209, 46014606.

(15) Wang, F. R.; Zhang, H. P.; Li, M. Z. Correlation Between Structure and Glass-Forming Ability in $\mathrm{Al}_{86} \mathrm{Ni}_{14-\mathrm{X}} \mathrm{la}_{\mathrm{x}}(\mathrm{X}=3,5,9)$ Alloys: an $\mathrm{Ab}$ Initio Molecular Dynamics Study. J. Alloys Compd. 2018, 763, 392-398.

(16) Bhattacharya, S. Kr.; Inama, F.; Scandoloab, S.; Supramolecular Charge Transfer Nanostructures. Phys. Chem. Chem. Phys. 2014, 16, 3103-3107.

(17) Sibik, J.; Elliott, S. R.; Zeitler, J. A. Thermal Decoupling of Molecular-Relaxation Processes from the Vibrational Density of States at Terahertz Frequencies in Supercooled Hydrogen-Bonded Liquids. J. Phys. Chem. Lett. 2014, 5, 1968-1972. 